\title{
Konsequenzen aus der gestiegenen Sensibilität gegenüber Jugendgewalt
}

Joachim Kersten

In Deutschland sinkt seit längerem die Zahl der jährlich registrierten Tötungsdelikte. Das ist kein schlechtes Zeichen. Die Anzeigen bei Taten der "Jugendgewalt“ hingegen steigen deutlich. Das erregt Besorgnis, aber alle seriösen Dunkelfeldstudien des letzten Jahrzehnts zeigen, dass es den allgemeinen Anstieg der von Jugendlichen begangenen Gewalttaten nicht gibt. Der Hellfeldanstieg beruht vermutlich auf der gestiegenen Anzeigebereitschaft, speziell gegenüber Tatverdächtigen, die „ausländisch“" erscheinen. Eine gesteigerte Sensibilität gegenüber Gewalt ist wiederum kein gesellschaftlicher Missstand. Für Praktiker in der Kriminalitätsbekämpfung bedeutet die gestiegene Anzeigbereitschaft allerdings mehr Arbeit. In der Mehrzahl verweisen Studien auch auf Probleme mit jugendlichen oder heranwachsenden Intensivtätern mit „Migrationshintergrund“. Sie stehen -wie die beiden Münchner Tatverdächtigen beim Überfall auf den Pensionär- im Zentrum von Kriminalitätsangst und sollen Forderungen nach härteren Strafen rechtfertigen. Früher habe ein „Ehrenkodex“ solche Gewalt verhindert meinen Nostalgiker in Politik und Pädagogik.

Der Rentner fiel einem realen Verhaltenskodex zum Opfer, dessen wichtigste Regeln in der Forschung von Elijah Anderson in einer Studie über die Slums von Philadelphia analysiert wurden: Ich klettere sozial nach oben, je besser ich jemand anderen runtermachen kann. Wenn jemand den Code der Straße nicht kennt und deshalb zu meinem Opfer wird, so ist er „selbst schuld“, Reue ist unnötig. Niemand sollte wagen, meine männliche Ehre anzurühren, denn sie ist das Einzige, was zählt. Nach außen muss klar sein: Nichts schreckt mich, ich schrecke vor nichts zurück. Dass ich nichts zu verlieren habe, schützt mich. Eine Gefängnisstrafe ist keine Katastrophe für meinesgleichen, es zählt wie eine bestandene Prüfung, sie steigert die Reputation. Außerdem sitzen meine Brüder schon dort, und wir halten zusammen und kontrollieren die Ökonomie hinter Gittern.

Der Kodex von Slumkulturen steuert das Miteinander von Personen und ihre Gewaltanwendung. Im Kern steht die Währung „Respekt“, ein Wort, dem einst eine Bedeutung von „Rücksicht“ zukam. Im Kodex des Kiez geht es jedoch um das genaue Gegenteil: Respekt wird als Tributleistung, als Zoll eingetrieben. Respekt verbindet sich dort wie in feudalen Zeiten mit einer Erwartung von Unterwürfigkeit. Man darf von niemandem ,gedissed“ werden. Dieser entscheidende Eskalationsanlass fehlt in fast allen wissenschaftlichen Konvoluten zur „Jugendgewalt“. Alltägliche Aggressivität hat andere Ursachen als nur immer die alte „Perspektiv- und Orientierungslosigkeit“, eine Erklärungsroutine der Bielefelder Gewaltforschung, die nur noch Gähnen auslöst.

Gewalt entsteht aus einer gesteigerten Empfindlichkeit gegenüber Andeutungen. Dissing wird als physischer Angriff aufgefasst und mit Aggression beantwortet. Zudem macht gemeinsame Gewalt auch noch Spaß. Das Opfer wird verweiblicht und dehumanisiert. Das hat schon Jack Katz' Analyse des Straßenraubs gezeigt. Genau das kennzeichnet auch die Gewalt der "nationalgesinnten “ Schlägergruppierungen in den neuen und alten Ländern.

Im Kiez bedeutet der Code eine Gewöhnung daran, dass der Glaube an Polizei und Justiz aufgegeben wurde. Die Streife kommt nicht, wenn man sie braucht, Polizei vertritt nur die Interessen der Mehrheit. Der Straßenkodex entsteht dort, wo der Einflussbereich der Polizei endet und wo es der persönlichen Verantwortung des Einzelnen unterliegt, sich um die eigene Sicherheit zu kümmern. Genau dies muss bekämpft werden und das hat mit längeren Strafen nichts zu tun. Das Auftreten unserer Polizeibeamten im Kiez muss zivilisiert, recht- und verhältnismäßig erfolgen. Ein professionelles, „anständiges“ Auftreten prägt insbesondere bei Kindern die Wahrnehmung von Fairness. Polizei ist im Kiez, stärker als bei „mündigen Bürgern “ - man ruft 110, weil der Rasenmäher des Nachbarn stört -, die „Visitenkarte der Gesellschaft".

Die Mehrheit der Leute in Problemvierteln will keine Abhängigkeit von staatlichen Hilfen, man ist bereit für den Nachwuchs Opfer zu bringen. Eltern sind streng, wachsam, religiös und kooperationsbereit. Wer Zuwanderer praktizierten moslemischen Glaubens unter einen Generalverdacht des islamistischen Terrors stellt, macht einen törichten Fehler.

Eine einflussreiche Minderheit hingegen nimmt im Kiez wenig Rücksicht auf andere. Als Eltern sind solche Personen selten oder gar nicht in der Lage, ihre eigenen Bedürfnisse mit denen ihrer Kinder zu vereinbaren. In einem Alltag voller Frustrationen und falscher Prioritäten haben Frauen verstrickte Beziehungen mit Männern, sie werden ausgebeutet und misshandelt. Verbitterung und Ärger sitzen tief, der versagte Wunsch nach Kontrolle führt zu einem unkontrollierten „Austeilen“, Sicherungen brennen schnell durch. Das ist die Basis des Zyklus der Gewaltbereitschaft, die sich über Generationen erstreckt: Kinder beobachten die Gewalt in der eigenen Wohnung. Die Grundregel wird erlernt, nach der Gewalt vor Recht geht. Härte ist gut.

Gelehrt wird die gewaltförmige Durchsetzung: Schlagen, verbales Herabsetzen, Beschimpfen, all dies wird Teil der Sozialisation. Wer Kämpfe für sich entscheiden kann, erntet den Respekt der anderen. Wenn dich jemand disst, mach' ihn fertig.

Auch die „anständigen“ Eltern bestrafen oder maßregeln ihre Kinder, wenn sie in Kämpfen unterliegen. So gibt es im Kiez eine Überschneidung von Erziehungsmethoden der „Bemühten“ und der Gewaltbereiten, schreibt Anderson.

„Angesagter" Stil und Selbstwert sind untrennbar verbunden: Kleidung, Schuhe, Schmuck sind Zeichen, Besitz, den niemand anrühren darf. Man kann allerdings auch durch den Raub von Dingen Zugehörigkeit erwerben. Die Tatverdächhtigen beim Münchner U-Bahn Vorfall und Hunderte andere Intensivtäter in unseren Städten haben dieses Wertsystem erlernt. Reue zeigen sie nur, wenn es ihnen der Anwalt nach der Verhaftung nahe legt. Tatsächlich beruht Reue auf Scham. Schuld ist für diese jungen Männer leichter zu ertragen als Scham. Das Unvermögen, sich zu schämen und Verantwortung für Schädigung zu übernehmen, wird durch ein höheres Strafmaß nicht beeinflusst. Auch nicht durch boot camps. Diesen Jugendlichen fehlt eine Fähigkeit zur Perspektivenübernahme. Von ihnen Reue zu erwarten, ist naiv. Eine langfristig angelegte Bekämpfung von Gewalt auf den Straßen und der schlimmeren, häufigeren Gewalt innerhalb der vier Wände, beide sind im Zuwanderungsmilieu sichtbarer als bei den sozial Abgesicherten, bedarf einer früheren und strikteren Konfrontation. Die Polizei ist zuständig, aber man kann ihr dies nicht überantworten. Wer Schaden verursacht, muss vor und mit anderen lernen, sich zu stellen und Empfindungen darüber zuzulassen. Das Beispiel der family conference an kanadischen oder neuseeländischen Schulen und in Jugendbehörden zeigt, dass solche „wiedereingliedernde Beschämung“ kein Pranger ist wie deutsche Pädagogen und Psychologen stets vermuten. Wer Gewalt verhindern will, muss ihre Neutralisierung im sozialen Umfeld bekämpfen. Das bedarf geschulter Kräfte, an deren Zahl und Ausbildung seit Jahren gespart wird. Vielleicht kann man die Geldstrafen der Liechtensteiner White Collar Bande für diesen Zweck einsetzen. 